\title{
Bond Valence Sum: A Powerful Tool for Determination of Oxidation States of Metal lons in Coordination Compounds
}

Tu N. Nguyen

Helen Scientific Research and Technological Development Co., Ltd, Ho Chi Minh City, Vietnam

Email: ngoctukhtn@gmail.com

\section{ABSTRACT}

Transition metal ions in coordination compounds can adopt different oxidation states. The Bond Valence Sum (BVS) model, based solely on structural information, relates the bond lengths around a metal center to its oxidation state. This model can provide details on the oxidation states of the metal ions and serves as an additional support for the accuracy of crystal structure determination. Herein, we introduce the fundamental concept of the BVS method and summarize the empirical BVS parameters method to mononuclear and polynuclear complexes will be discussed.

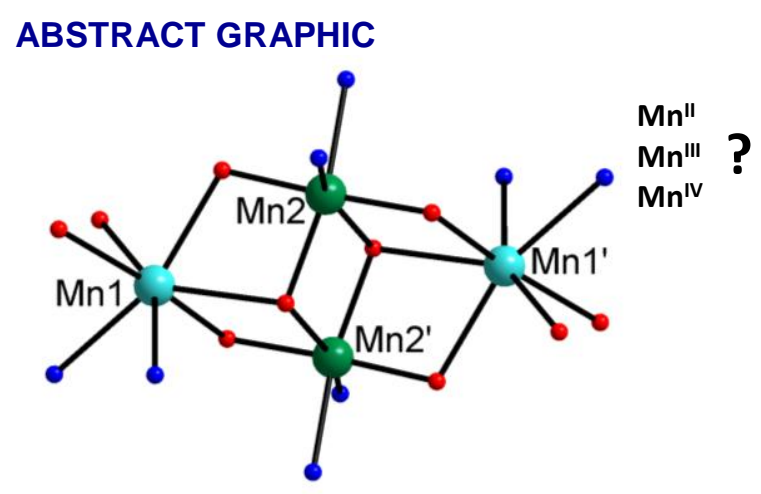

\section{KEYWORDS} Computer-Based Learning. 


\section{INTRODUCTION}

The Bond Valence Sum (BVS) method has been used by several research groups to estimate the oxidation states of metal ions in coordination compounds. The original concept of BVS was developed from Pauling's rules, ${ }^{1}$ which allow for predicting and rationalizing the crystal structures of ionic compounds. Pauling's second rule defines the valence $(s)$ of the bond $\mathrm{M}-\mathrm{X}$ in compound $\mathrm{MX}_{\mathrm{n}}$ as the ratio between the charge and coordination number of the central atom $\mathrm{M}$. The local electroneutrality in a stable ionic structure must be preserved, i.e. the sum of the bond valences around each atom should equal its oxidation state. Later, Pauling realized that a cation and its neighboring anion shorten their relative ionic radii when electronic density between the two species is increased. ${ }^{2}$ This represents the first attempt to relate the length of a bond to its strength. In 1970, the direct correlation between valence and bond length was deduced by Donnay and Allmann ${ }^{3}$ and confirmed by Brown and Shannon in 1973 when they studied a large number of metal-oxide inorganic compounds. ${ }^{4}$ Many attempts to establish a function for the prediction of the valence through the sum of all the bond lengths were reported, but it was not until 1985 when Brown and Altermatt proposed a general expression shown in equation 1,5

$$
V_{\mathrm{i}}=\sum s_{\mathrm{ij}}=\sum \exp \left[\left(R_{0^{-}} r_{\mathrm{ij}}\right) / b\right]
$$

where $V_{1}$ is the oxidation state of atom $\mathrm{i} ; s_{\mathrm{ij}}$ and $r_{\mathrm{ij}}$ are the valence and the length of the bond between atoms $\mathrm{i}$ and $\mathrm{j}$, respectively; $R_{0}$ is the empirically determined distance for a given cation-anion pair; $b$ is the "universal parameter" 6 and is set equal to $0.37 .{ }^{7} \mathrm{~V}$ is the sum of all valences $s_{\mathrm{ij}}$ of a coordination sphere around the metal ion, and its value is the approximation of the formal oxidation state. For instance, by applying the BVS to 45 a metal ion in the +3 oxidation state, the sum of all its bond valences will lead to a value close to the integer three. The same calculation applied with $R_{0}$ parameters for 
other oxidation states will also provide a $V$ value close to the integer three, but with a larger discrepancy. This model can be applied to any coordination environment

Table 1. Bond valence parameters $R_{0}$ for $3 d$ transition metal ions.

\begin{tabular}{|c|c|c|c|c|c|c|c|c|}
\hline & $\begin{array}{l}\text { Ox. } \\
\text { No }\end{array}$ & 0 & $\mathrm{~F}$ & $\mathrm{Cl}$ & $\mathrm{N}$ & $\mathrm{Br}$ & I & $\mathrm{S}$ \\
\hline \multirow[t]{3}{*}{$\mathrm{Ti}$} & 2 & & 2.15 & 2.31 & & 2.49 & & \\
\hline & 3 & 1.791 & 1.723 & 2.17 & & & 2.52 & 2.11 \\
\hline & 4 & 1.815 & 1.76 & 2.19 & & 2.36 & & 2.29 \\
\hline \multirow[t]{4}{*}{ V } & 2 & 1.70 & 2.16 & 2.44 & & & & 2.11 \\
\hline & 3 & 1.743 & 1.702 & 2.19 & 1.813 & 2.33 & & 2.185 \\
\hline & 4 & 1.784 & 1.70 & 2.16 & 1.875 & & & 2.226 \\
\hline & 5 & 1.803 & 1.71 & 2.16 & & & & 2.25 \\
\hline \multirow[t]{5}{*}{$\mathrm{Cr}$} & 2 & 1.73 & 1.67 & 2.09 & & 2.26 & 2.48 & \\
\hline & 3 & 1.724 & 1.657 & 2.08 & 1.81 & 2.28 & & 2.162 \\
\hline & 4 & 1.81 & 1.56 & & & & & \\
\hline & 5 & 1.76 & & & & & & \\
\hline & 6 & 1.794 & 1.74 & 2.12 & & & & \\
\hline \multirow[t]{3}{*}{$\mathrm{Mn}$} & 2 & 1.765 & 1.698 & 2.133 & 1.849 & 2.34 & 2.52 & 2.22 \\
\hline & 3 & 1.732 & 1.66 & 2.14 & 1.837 & & & \\
\hline & 4 & 1.75 & 1.71 & 2.13 & 1.822 & & & \\
\hline \multirow[t]{2}{*}{$\mathrm{Fe}$} & 2 & 1.734 & 1.65 & 2.06 & 1.769 & 2.21 & 2.47 & 2.125 \\
\hline & 3 & 1.759 & 1.679 & 2.09 & 1.815 & 2.22 & & 2.149 \\
\hline \multirow[t]{2}{*}{ Co } & 2 & 1.685 & 1.64 & 2.033 & 1.65 & & & 1.94 \\
\hline & 3 & 1.637 & 1.62 & 2.05 & 1.75 & & & 2.02 \\
\hline \multirow[t]{2}{*}{$\mathrm{Ni}$} & 2 & 1.675 & 1.596 & 2.02 & 1.647 & 2.20 & 2.40 & 1.937 \\
\hline & 3 & 1.75 & 1.58 & 1.731 & & & & 2.040 \\
\hline \multirow[t]{2}{*}{$\mathrm{Cu}$} & 1 & 1.504 & 1.60 & 1.858 & 1.61 & 2.03 & 2.155 & 1.811 \\
\hline & 2 & 1.655 & 1.594 & 2.00 & 1.713 & 2.134 & 2.36 & 2.024 \\
\hline
\end{tabular}

Table 2. Bond valence parameters $\boldsymbol{R}_{0}$ for selective $4 \mathrm{~d}$ transition metal ions.

\begin{tabular}{|c|c|c|c|c|c|c|c|c|}
\hline & $\begin{array}{l}\text { Ox. } \\
\text { No }\end{array}$ & 0 & F & $\mathrm{Cl}$ & $\mathrm{N}$ & $\mathrm{Br}$ & I & $S$ \\
\hline \multirow[t]{2}{*}{$\mathrm{Zr}$} & 2 & 2.34 & 2.24 & 2.58 & & & & \\
\hline & 4 & 1.937 & 1.854 & 2.33 & 2.11 & 2.48 & 2.69 & 2.41 \\
\hline \multirow[t]{3}{*}{$\mathrm{Nb}$} & 3 & & 1.71 & 2.20 & & 2.35 & & \\
\hline & 4 & 1.88 & 1.90 & 2.236 & 2.004 & 2.62 & & \\
\hline & 5 & 1.911 & 1.87 & 2.27 & & & 2.77 & \\
\hline \multirow[t]{4}{*}{ Mo } & 3 & 1.834 & & 2.22 & 1.96 & 2.34 & & \\
\hline & 4 & 1.856 & 1.80 & & 2.043 & & & 2.235 \\
\hline & 5 & 1.878 & & 2.26 & 2.009 & & & 2.288 \\
\hline & 6 & 1.907 & 1.81 & 2.28 & 2.009 & & & 2.331 \\
\hline \multirow[t]{2}{*}{$\mathrm{Tc}$} & 4 & 1.841 & & 2.21 & & & & \\
\hline & 5 & 1.859 & & & & & & \\
\hline \multirow[t]{2}{*}{$\mathrm{Ru}$} & 3 & 1.77 & 2.12 & 2.25 & & & & \\
\hline & 4 & 1.834 & 1.74 & 2.21 & & & & 2.21 \\
\hline
\end{tabular}


regardless of the coordination number. In fact, a metal ion with the same oxidation state will generally display longer bonds with an increase in coordination number. The empirical values of $R_{0}$ have been calculated and reported for many metal-ligand combinations and different oxidation states. ${ }^{8,9,10}$ Table 1 and 2 summarize the recommended $R_{0}$ values for selective $3 \mathrm{~d}$ and $4 \mathrm{~d}$ metal ions that can have more than one oxidation state and are in combination with common ligand atoms $\mathrm{O}, \mathrm{F}, \mathrm{Cl}, \mathrm{N}, \mathrm{Br}, \mathrm{I}$, and S. A full table of $R_{0}$ values can be found in reference 10 .

In practice, BVS calculations can be used to determine the oxidation states of metal ions in a complex before finalizing its X-ray crystal structure or to check the accuracy of a completed structure. Applications of the BVS model to mononuclear and polynuclear complexes will be discussed as follows.

\section{BVS CALCULATIONS FOR MONONUCLEAR COMPLEXES}

For mononuclear complexes, the oxidation state can be deduced from the physical properties of the metal ions such as magnetic moment, chemical shift, or isomeric shift. Measurements of these properties might require sophisticated instrumentation that might not be easily accessed by students. In the two examples below, we present a simple method using the BVS model to obtain the oxidation state of the metal ions. Example 1: A vanadium complex

In 2013, Szymczak and coworkers reported the X-ray crystal structure of a vanadium complex $\left[\mathrm{V}\left(\mathrm{Terpy}^{\mathrm{Me}_{2}}\right) \mathrm{Cl}_{3}\right](\mathbf{1})$, which is shown in Figure 1. No counter anion was found, thus the $\mathrm{V}$ atom has the oxidation state $3+$. The authors did not perform the BVS calculation. Instead, they confirmed the oxidation state of the V ion by measuring the magnetic moment of the complex, which gave a value of $2.63 \mu_{\mathrm{B}}$, supporting a $\mathrm{d}^{2} \mathrm{VIII}$ electron configuration. ${ }^{11}$

To provide additional support for this finding and eliminate the possibility of the presence of a more common VIV ion, the BVS calculation was performed with the V-N 
and $\mathrm{V}-\mathrm{Cl}$ bond lengths obtained from the X-ray crystal structure, $R_{0}$ values extracted from Table 1 for VIII and VIV, and $b$ is set equal to 0.37. An example of the calculation of the valence for the bond V1-Cl1 (bond length $r_{\mathrm{V} 1-\mathrm{Cl1}}=2.3196 \AA$ ) is as follows:

For the case of VIII, $R_{0}=2.19, s=\exp \left[\left(R_{0}-r_{\mathrm{V} 1-\mathrm{Cl} 1}\right) / b\right]=0.70$

For the case of VIV $, R_{0}=2.16, s=\exp \left[\left(R_{0}-r_{\mathrm{V} 1-\mathrm{Cl} 1}\right) / b\right]=0.65$

Similar calculations can be done for other vanadium-ligand bonds. The sum of the valences of the bonds around the $\mathrm{V}$ ion will provide an estimation of its oxidation state. The result of the BVS calculations is summarized in Table 3. The sum of bond valences is 3.16 for $\mathrm{V}^{\mathrm{III}}$ and 3.23 for $\mathrm{VIV}^{\mathrm{IV}}$; these values are closest to the integer three. Thus, we agree with the authors that the vanadium ion in complex $\mathbf{1}$ has a +3 oxidation state.

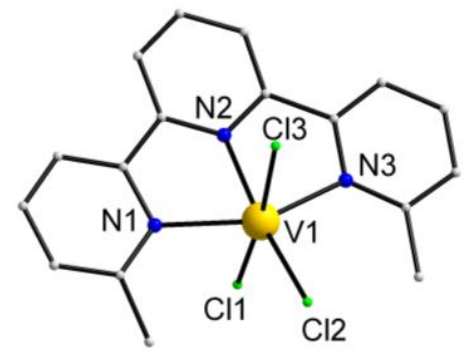

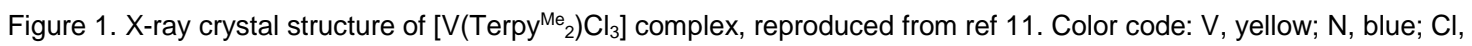
green; C, gray. Hydrogen atoms have been omitted for clarity.

Table 3. Bond valence sum for the $V$ ion in complex 1.

\begin{tabular}{|c|c|c|c|}
\hline \multirow[t]{2}{*}{ Bond } & \multirow[t]{2}{*}{ Bond length $(\AA)$} & \multicolumn{2}{|c|}{ Valence } \\
\hline & & VIII & VIV \\
\hline V1-Cl1 & 2.3196 & 0.70 & 0.65 \\
\hline V1-Cl2 & 2.3274 & 0.69 & 0.64 \\
\hline V1-Cl3 & 2.3956 & 0.57 & 0.53 \\
\hline V1-N1 & 2.1936 & 0.36 & 0.42 \\
\hline V1-N2 & 2.0720 & 0.50 & 0.59 \\
\hline V1-N3 & 2.2117 & 0.34 & 0.40 \\
\hline \multicolumn{2}{|c|}{ Sum } & 3.16 & 3.23 \\
\hline
\end{tabular}

\section{Example 2: Two molybdenum complexes}

Sugimoto and co-workers presented two molybdenum mononuclear complexes as the synthetic model for molybdenum cofactors. ${ }^{12}$ The complexes are assigned as 2 and 
3 shown in Figure 2 and they possess Mo ions with oxidation state +5 and +6 , respectively. The authors did not perform BVS calculations on $\mathbf{2}$ and $\mathbf{3}$.

To validate the accuracy of the structure determination, BVS calculations with $R_{0}$ values for $\mathrm{Mo}^{\mathrm{V}}$ and $\mathrm{Mo}^{\mathrm{VI}}$ were performed and summarized in Table 4. If the calculated and postulated values differ markedly, the crystal structure assignment is likely incorrect. As shown in Table 4, the obtained BVS values for complexes 2 and $\mathbf{3}$ are 4.98 and 5.76 , which match pretty well to the oxidation states of +5 and +6 of the Mo ions. It is worth noting that the presence of disorders is one of the reasons for increasing the discrepancy between the BVS value and the postulated oxidation state.
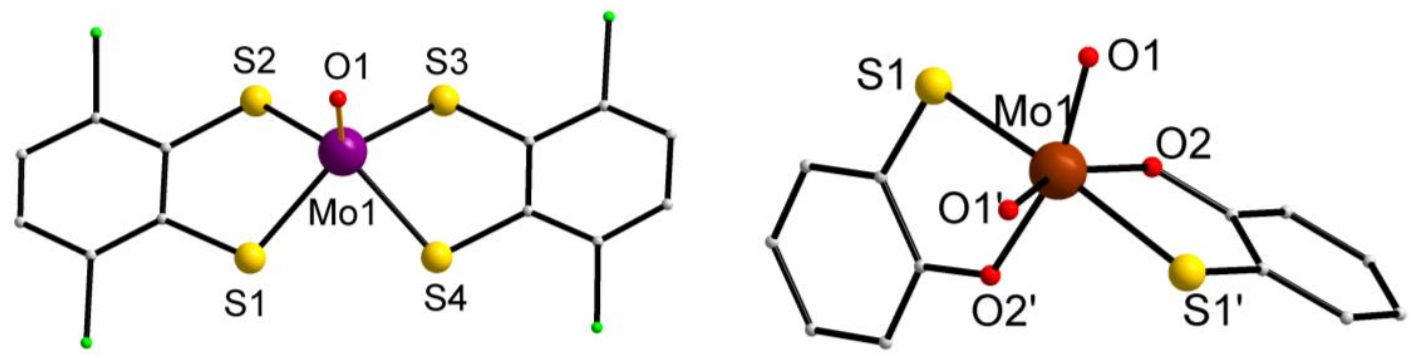

Figure 2. X-ray crystal structures of a $\mathrm{Mo}^{\mathrm{V}}$ (left) (2) and a $\mathrm{Mo}^{\mathrm{VI}}$ (right) (3) complex, reproduced from ref 12. Color code: $\mathrm{Mo}^{\mathrm{v}}$, violet; $\mathrm{Mo}^{\mathrm{VI}}$, brown; S: gold; O, red, Cl, green; C, gray. Hydrogen atoms have been omitted for clarity. The prime presents the crystallographic symmetry.

Table 4. Bond valence sum for the Mo ions in complexes 2 (left) and 3 (right).

\begin{tabular}{lcc|ccc} 
Bond & Bond length $(\AA)$ & Valence & Bond & Bond length $(\AA)$ & Valence \\
\hline Mo1-01 & 1.6560 & 1.82 & Mo1-01 & 1.7396 & 1.57 \\
Mo1-S1 & 2.4075 & 0.72 & Mo1-01' & 1.7396 & 1.57 \\
Mo1-S2 & 2.3202 & 0.92 & Mo1-02 & 2.0705 & 0.64 \\
Mo1-S3 & 2.3533 & 0.84 & Mo1-02' & 2.0705 & 0.64 \\
Mo1-S4 & 2.4330 & 0.68 & Mo1-S1 & 2.4807 & 0.67 \\
& & & Mo1-S1' & 2.4807 & 0.67 \\
& Sum & 4.98 & \multicolumn{2}{|c}{ Sum } \\
\hline
\end{tabular}

\section{BVS CALCULATIONS FOR POLYNUCLEAR COMPLEXES}

X-ray crystal structures of polynuclear complexes are more complicated than those of mononuclear ones since the metal ions can adopt different oxidation states and 
finding the counter ions to balance the charge of the molecule is often a challenging task for crystallographers. For molecules that have mixed-valence ions, the physical properties are contributed from all ions; thus, physical measurements are not always as helpful as for mononuclear complexes. BVS calculations on each metal ion will greatly help to improve the X-ray structure refinement.

\section{Example 3: A tetranuclear manganese complex}

Clérac, Miyasaka, and coworkers reported a manganese complex containing four Mn ions in an attempt to synthesize new single-molecule magnets. ${ }^{13}$ The structure of the complex $\left[\mathrm{Mn}_{4}(\mathrm{hmp})_{6}\left(\mathrm{CH}_{3} \mathrm{CN}\right)_{2}\left(\mathrm{H}_{2} \mathrm{O}\right)_{4}\right]\left(\mathrm{ClO}_{4}\right)_{4}(\mathbf{4})$ is shown in Figure 3. $\mathrm{Mn} 1$ and $\mathrm{Mn} 2$ were assigned as $\mathrm{Mn}^{\mathrm{II}}$ and $\mathrm{Mn}^{\mathrm{III}}$, respectively. The authors were able to fit the magnetic susceptibility data to the model of a $\mathrm{Mn}_{2}{ }_{2} \mathrm{Mn}^{\mathrm{III}}{ }_{2}$ butterfly core. No BVS calculation was reported for complex 4 .

To estimate the oxidation state of Mn1 and Mn2 using the BVS model, the sum of valences were calculated using the $R_{0}$ values for $\mathrm{Mn}^{\mathrm{II}}, \mathrm{Mn}^{\mathrm{III}}$, and $\mathrm{Mn}^{\mathrm{IV}}$ that can be obtained in Table 1. The integer number that is closest to the BVS values is the oxidation state of the ion. As shown in Table 5, Mn1 and Mn2 with BVS values of $\sim 2$ and $\sim 3$ have the expected +2 and +3 oxidation states, respectively.

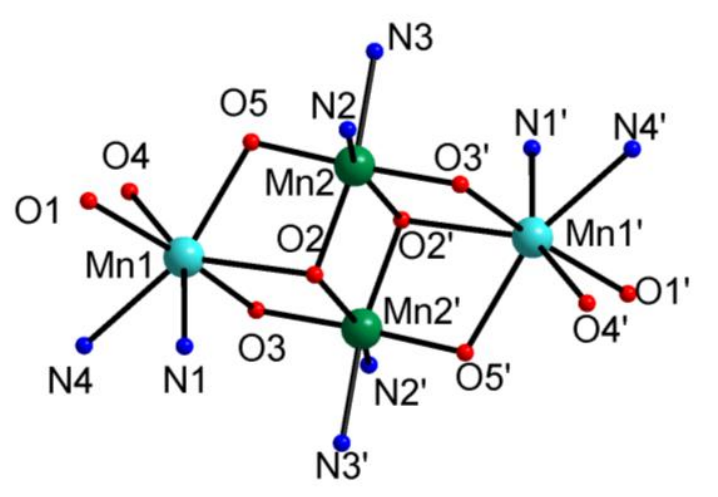

Figure 3. X-ray crystal structures of the core of complex 4, reproduced from ref 13. Color code: $\mathrm{Mn}^{\prime \prime \prime}$, green; $\mathrm{Mn}$ ", aqua; O, red; $\mathrm{N}$, blue. Hydrogen atoms have been omitted for clarity. The prime presents the crystallographic symmetry. 
Table 5. Bond valence sum for the Mn ions in complex 4.

\begin{tabular}{cccccc} 
& Bond & Bond length $(\AA)$ & \multicolumn{3}{c}{ Valence } \\
& & & Mn & Mn & Mn \\
\hline Mn1 & Mn1-01 & 2.1931 & 0.31 & 0.29 & 0.30 \\
& Mn1-02 & 2.3712 & 0.19 & 0.18 & 0.19 \\
& Mn1-03 & 2.1761 & 0.33 & 0.30 & 0.32 \\
& Mn1-04 & 2.2389 & 0.28 & 0.25 & 0.27 \\
& Mn1-05 & 2.2472 & 0.27 & 0.25 & 0.26 \\
& Mn1-N1 & 2.3361 & 0.27 & 0.26 & 0.25 \\
Mn1-N4 & 2.3692 & 0.25 & 0.24 & 0.23 \\
& & & 1.90 & 1.77 & 1.82 \\
& Mn2-02 & 1.9581 & 0.59 & 0.54 & 0.57 \\
Mn2-02' & 2.2435 & 0.27 & 0.25 & 0.26 \\
Mn2-03 & 1.8748 & 0.74 & 0.68 & 0.71 \\
Mn2-05 & 1.8601 & 0.77 & 0.71 & 0.74 \\
Mn2-N2 & 2.1902 & 0.40 & 0.38 & 0.37 \\
Mn2-N3 & 2.0450 & 0.59 & 0.57 & 0.55 \\
& & 3.36 & 3.13 & 3.20 \\
\hline
\end{tabular}

Example 4: A Zr6 $\mathrm{Co}_{6}$ complex

Kögerler and coworkers reported a bimetallic complex (5) containing six Co and six Zr ions. ${ }^{14}$ As shown in Figure 4, the core of complex $\mathbf{5}$ has a C6 axis, thus all the Co and $\mathrm{Zr}$ ions are equivalent upon applying a 6-fold rotation. Without performing BVS calculations, the authors assigned the Co and $\mathrm{Zr}$ ions to their most common oxidation states, which are +2 for Co and +4 for $\mathrm{Zr}$. We can confirm this by applying BVS calculations for both ions as displayed in Table 6.

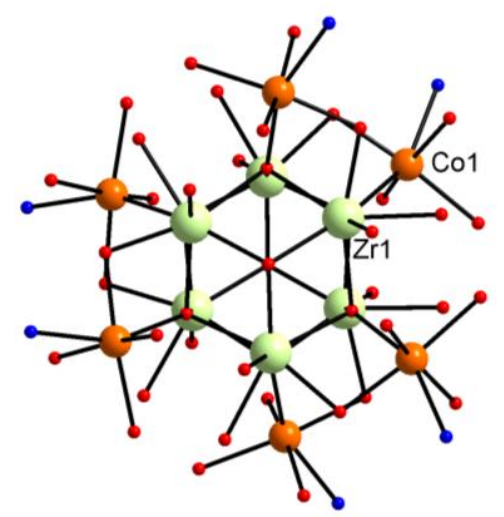

140 Figure 4. X-ray crystal structures of the core of complex 5, reproduced from ref 14. Color code: Co, orange; Zr, light green; $\mathrm{O}$, red; $\mathrm{N}$, blue. Hydrogen atoms have been omitted for clarity. 
Table 6. Bond valence sum for the $\mathrm{Zr}$ and Co ions in complex 5.

\begin{tabular}{lccccc} 
Bond & Bond length $(\AA)$ & Valence & Bond & Bond length $(\AA)$ & Valence \\
\hline Zr1-01 & 2.1090 & 0.63 & Co1-01 & 2.1891 & 0.26 \\
Zr1-01' & 2.1514 & 0.56 & Co1-05 & 2.0223 & 0.40 \\
Zr1-01" & 2.1930 & 0.50 & Co1-06 & 2.1073 & 0.32 \\
Zr1-02 & 2.1047 & 0.64 & Co1-07 & 2.0810 & 0.34 \\
Zr1-03 & 2.1671 & 0.54 & Co1-08 & 2.0779 & 0.35 \\
Zr1-04 & 2.2284 & 0.45 & Co1-N1 & 2.1886 & 0.23 \\
Zr1-05 & 2.1270 & 0.60 & \multicolumn{3}{l}{ Sum } \\
\multicolumn{7}{c}{ Sum } & 3.92 & \multicolumn{3}{l}{1.90} \\
\hline
\end{tabular}

\section{CONCLUSION}

The BVS is a relatively simple calculation that can be carried out for many oxidation states of metal ions has been demonstrated for both mononuclear and polynuclear complexes. Although BVS has not been applied routinely, we hope this work will encourage students to begin the habit of using this tool to obtain a better understanding of their interested coordination complexes.

\section{REFERENCES}

1. Pauling, L. The Principles Determining The Structure of Complex Ionic Crystals. J. Am. Chem.Soc. 1929, 51, 1010.

2. Pauling, L. Atomic Radii and Interatomic Distances in Metals. J. Am. Chem. Soc. 1947, 69, 542.

3. Donnay, G.; Allmann, R. How to Recognize $\mathrm{O}^{2-}, \mathrm{OH}^{-}$, and $\mathrm{H}_{2} \mathrm{O}$ in Crystal Structures Determined by X-rays. Am. Mineral. 1970, 55, 1003.

4. Brown, I. D.; Shannon, R. D. Empirical Bond-Strength-Bond-Length Curves for Oxides. Acta Crystallogr. A. 1973, A29, 266.

5. Brown, I. D.; Altermatt, D. Bond-Valence Parameters Obtained from a Systematic Analysis of the Inorganic Crystal Structure Database. Acta Crystallogr. B. 1985, B41, 244. 
6. Garcia-Rodriguez, L.; Rute-Perez, A.; Pinero, J. R.; Gonzalez-Silgo, C. Bond-Valence Parameters for Ammonium-Anion Interactions. Acta Crystallogr. B. 2000, B56, 565.

7. Brown, I. D. Chemical and Steric Constraints in Inorganic Solids. Acta Crystallogr. B. 1992, B48, 553.

8. Brese, N. E.; O’Keeffe, M. Bond-Valence Parameters for Solids. Acta Crystallogr. B. 1991, B47, 192.

9. O’Keeffe, M. A Method Calculating Bond Valences in Crystals. Acta Crystallogr. A. 1990, A46, 138.

10. Brown, I. D. Bond Valence Parameters. http://www.iucr.org/resources/data/datasets/bondvalence-parameters (accessed Jun 30, 2015).

11. Tutusaus, O.; Ni, C.; Szymczak, N. K. A Transition Metal Lewis Acid/Base Triad System for Cooperative Substrate Binding. J. Am. Chem. Soc. 2013, 135, 3403

12. Sugimoto, H.; Tarumizu, M.; Tanaka, K.; Miyakea, H.; Tsukube, H. A New Series of Molybdenum-(IV), -(V), and -(VI) Dithiolate Compounds as Active Site Models of Molybdoenzymes: Preparation, Crystal structures, Spectroscopic/Electrochemical Properties and Reactivity in Oxygen Atom Transfer. Dalton Trans. 2005, 3558.

13. Lecren, L.; Li, Y-G.; Wernsdorfer, W.; Roubeau, O.; Miyasaka, H.; Clérac, R. $\left[\mathrm{Mn}_{4}(\mathrm{hmp})_{6}\left(\mathrm{CH}_{3} \mathrm{CN}\right)_{2}\left(\mathrm{H}_{2} \mathrm{O}\right)_{4}\right]^{4+}$ : A New Single-Molecule Magnet with the Highest Blocking Temperature in the Mn4/hmp Family of Compounds. Inorg. Chem. Commun. 2005, 8, 626.

14. Malaestean, I. L.; Alıc1, M. K.; Besson, C.; Ellernb, A.; Kögerler, P. Solid-State Coexistence of $\left\{\mathrm{Zr}_{12}\right\}$ and $\left\{\mathrm{Zr}_{6}\right\}$ Zirconium Oxocarboxylate Clusters. Cryst. Eng. Comm, 2014, 16, 43. 